\title{
Mechanical Response of Subloading Surface Model with Tangential Plasticity
}

\author{
Seiichiro Tsutsumi*, Koichi Hashiguchi**, Takashi Okayasu***, Koshiro Saitoh*, Masaya Sugimoto**** \\ * M. of Agr., Dept. of Agr. Eng., Kyushu University, Hakozaki, Higashi-ku, Fukuoka 812-8581 \\ ** Dr. of Eng. and Dr. of Agr., Professor, Dept. of Agr. Eng., Kyushu University, Hakozaki, Higashi-ku, Fukuoka $812-8581$ \\ *** Dr. of Agr., Researcher, Dept. of Agr. Eng., Kyushu University, Hakozaki, Higashi-ku, Fukuoka 812-8581 \\ **** B. of Agr., Dept. of Agr. Eng., Kyushu University, Hakozaki, Higashi-ku, Fukuoka 812-8581
}

\begin{abstract}
The mechanical response of the elastoplastic constitutive model for soils is examined, which is based on the subloading surface model with tangential stretching describing the inelastic stretching induced by the stress rate component tangential to the yield/loading surface. The stress rate response to the imposed stretching under triaxial condition in not only hardening but also softening states on the meridian and the deviatoric planes is shown firstly. Then, it is indicated that the stress rate response in the deviatoric stress rate plane is decreased to the tangential direction of subloading surface by the tangential stretching effect. Further, a nose is predicted in the response envelope to the imposed stretching inducing the axisymmetric deformation and the response envelope in the softening state exhibits a crescent form.
\end{abstract}

Key Words: elastoplastic constitutive equation, subloading surface model, tangential stress rate

\section{Introduction}

In the traditional elastoplastic constitutive equation with a single smooth plastic potential surface, the plastic stretching is independent of the stress rate component tangential to the yield/loading surface, called the tangential stress rate, and then the direction of the plastic stretching is given uniquely to the normal direction of a plastic potential surface. The disregard of the tangential stress rate effect leads to the prediction of unrealistically stiff response of materials when a loading path deviates from the proportional loading as observed in the localization of deformation. The tangential stress rate effect has been experimentally observed for soils ${ }^{1), 2), 3)}$.

As reviewed previously by the authors ${ }^{4), 5)}$, various elastoplastic constitutive equations extended to describe the tangential stress rate effect have been proposed up to the present ${ }^{5), 6), 7,8)}$. Among them, however, only the subloading surface model with tangential stress rate effect ${ }^{5)}$ would be applicable to a general loading process for materials with an arbitrary yield/loading surface. The model fulfills the mechanical requirements for constitutive equations ${ }^{9), 10), 11), 12)}$, i.e. the continuity condition, the smoothness condition, the Masing effect and the work rate-stiffness relaxation. It is formulated by introducing the additional stretching, named tangential stretching induced by the deviatoric tangential stress rate, into the subloading surface model ${ }^{13), 14), 15), 16)}$ exhibiting smooth elastic-plastic transition. It is of simple form of rate-linearity enabling the reciprocal expression, i.e. the analytical expression of stretching in terms of stress rate and its inverse expression, and keeps the symmetry of the stiffness modulus, and thus leading to the convenience in the analyses of boundary value problems.

Some attempts ${ }^{8,17), 18)}$ to search the mechanical responses of elastoplastic constitutive models of soils have been conducted by imposing stress rates or stretchings to various directions (stress probes). Note that the mechanical responses of the subloading surface model with tangential stretching applied for metals was examined by the authors ${ }^{5}$, whilst the analysis was conducted for a plastically-incompressible metals exhibiting only a hardening behavior under the imposed stress rate condition in the deviatoric stress rate plane.

In this article, the subloading surface model with tangential stretching is applied for soils with the isotropic hardening rule, so that the mechanical responses of the model for soils could be understandable clearly in not only normal-consolidated but also over-consolidated states. Then, the basic characteristics of the model are examined by calculating the stress rate response to the imposed stretching in not only hardening but also 
softening states.

The tensile stress (rate) and stretching (a symmetric component of velocity gradient) are taken to be positive throughout in this article.

\section{Outline of the Subloading Surface Model with Tangential Stretching}

In this chapter, the subloading surface model with tangential stretching ${ }^{5)}$ previously proposed by the authors is reviewed briefly.

\subsection{The extended subloading surface model}

Denoting the current configuration of material particle as $\boldsymbol{x}$ and the current velocity as $v$, the velocity gradient is described as $\boldsymbol{L}=\partial \boldsymbol{v} / \partial \boldsymbol{x}$, by which the stretching is defined as $\boldsymbol{D}$ $\left(=\left(\boldsymbol{L}+\boldsymbol{L}^{\mathrm{T}}\right) / 2\right),()^{\mathrm{T}}$ standing for the transpose.

Now let it be assumed that the stretching $D$ is additively decomposed into the elastic stretching $D^{e}$ and the plastic stretching $\boldsymbol{D}^{P}$, i.e.

$$
\boldsymbol{D}=\boldsymbol{D}^{e}+\boldsymbol{D}^{P},
$$

where the elastic stretching $D^{e}$ is given by

$$
D^{e}=E^{-1} \stackrel{\circ}{\sigma} \text {. }
$$

$\sigma$ is the Cauchy stress tensor and $\left(^{\circ}\right)$ indicates the proper corotational rate with the objectivity ${ }^{19,20)}$ and the fourth-order tensor $\boldsymbol{E}$ is the elastic modulus.

Assume the yield condition:

$$
f(\hat{\boldsymbol{\sigma}}, \boldsymbol{H})=F(H),
$$

where

$$
\hat{\sigma} \equiv \boldsymbol{\sigma}-\boldsymbol{\alpha} .
$$

The second-order tensor $\boldsymbol{\alpha}$ is the reference point on or inside the yield surface, which plays the role of the kinematic hardening variable as it translates with the plastic deformation. The scalar $H$ and the second-order tensor $\boldsymbol{H}$ are the isotropic and the anisotropic hardening variables, respectively. Let it be assumed that the function $f$ is homogeneous of degree one in the tensor $\hat{\sigma}$

Hereinafter, the elastoplastic constitutive equation will be formulated in the framework of the unconventional plasticity ${ }^{22)}$ defined as the extended plasticity theory such that the interior of the yield surface is not a purely elastic domain but a plastic deformation is induced by the rate of stress inside the yield surface. Thus, the conventional yield surface is renamed as the normal-yield surface, since its interior is not regarded as a purely elastic domain in the present model.

Now, the subloading surface ${ }^{14), 15)}$ is introduced, which always passes through the current stress point $\sigma$ and also keeps the similar shape to the normal-yield surface and the positioning of similarity to the normal-yield surface with

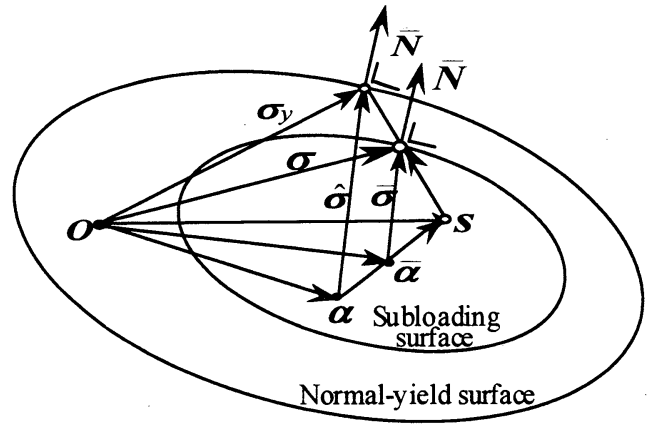

Fig. 1. Normal-y ield and subloading surfaces

respect to the similarity-center $s$. Here, note that the similarity-center has to lie inside both the normal-yield and subloading surfaces, since these surfaces are not allowed to intersect each other, whilst the subloading surface plays the role of loading surface. The approaching degree to the normal-yield state can be described by the ratio of the size of the subloading surface to that of the normal-yield surface, i.e. the similarity-ratio $R$ of these surfaces. Hereinafter, let the similarity-ratio $R$ be called the normal-yield ratio. Then, it holds that

$$
\sigma_{y}=\frac{1}{R}\{\sigma-(1-R) s\} \quad\left(\boldsymbol{\sigma}-\boldsymbol{s}=R\left(\sigma_{y}-s\right)\right),
$$

where $\sigma_{y}$ on the normal-yield surface is the conjugate stress of the current stress on the subloading surface (see Fig. 1).

By substituting Eq. (5) into Eq. (4) with regarding $\sigma$ in Eq. (4) as $\sigma_{y}$, the subloading surface is described as

$$
f(\bar{\sigma}, \boldsymbol{H})=R F(H),
$$

where

$$
\begin{aligned}
& \overline{\boldsymbol{\sigma}} \equiv \boldsymbol{\sigma}-\overline{\boldsymbol{\alpha}}\left(=R\left(\sigma_{y}-\boldsymbol{\alpha}\right)\right), \\
& \overline{\boldsymbol{\alpha}} \equiv \boldsymbol{s}-R(\boldsymbol{s}-\boldsymbol{\alpha}) \quad(\overline{\boldsymbol{\alpha}}-\boldsymbol{s}=R(\boldsymbol{\alpha}-\boldsymbol{s})) .
\end{aligned}
$$

$\overline{\boldsymbol{\alpha}}$ on or inside the subloading surface is the conjugate point of $\boldsymbol{\alpha}$ on or inside the normal-yield surface. In calculation, $R$ is first determined from Eq. (6) with Eqs. (7) and (8) by substituting values of $\boldsymbol{\sigma}, H, \boldsymbol{\alpha}, \boldsymbol{H}$ and $\boldsymbol{s}$, and thereafter $\overline{\boldsymbol{\alpha}}$ is found from Eq. (8).

It is observed from experiments that the stress asymptotically approaches to the normal-yield surface in the plastic loading process $\boldsymbol{D}^{P} \neq \boldsymbol{0}$. Thus, let the following evolution equation of the normal-yield ratio $R$ be assumed.

$$
\dot{R}=U\left\|D^{p}\right\| \text { for } \boldsymbol{D}^{p} \neq \boldsymbol{0},
$$

where $U$ is the monotonically decreasing function of the normal-yield ratio $R$, satisfying

$$
\begin{gathered}
U= \begin{cases}\infty & \text { for } R=0, \\
0 & \text { for } R=1,\end{cases} \\
(U<0 \text { for } R>1) .
\end{gathered}
$$

( ) stands for the material-time derivative and \|\| the magnitude. Let the function $U$ satisfying $\mathrm{Eq}$. (10) be simply 
given by

$$
U=-u_{R} \ln R,
$$

where $u_{R}$ is a material constant prescribing the approaching rate of the current stress to the normal-yield surface with a plastic deformation.

The similarity-center $s$ is required to translate with the plastic deformation in order to describe realistically the cyclic loading behavior exhibiting the Masing effect ${ }^{10), 23), 24)}$. The translation rule of $s$ is given as

$$
\begin{aligned}
\stackrel{\circ}{\boldsymbol{s}}= & c_{\boldsymbol{S}}\left\|\boldsymbol{D}^{p}\right\| \frac{\tilde{\boldsymbol{\sigma}}}{R}+\stackrel{\circ}{\boldsymbol{\alpha}} \\
& +\frac{1}{F}\left\{F^{\prime} \stackrel{\bullet}{H}-\operatorname{tr}\left(\frac{\partial f(\hat{\boldsymbol{s}}, \boldsymbol{H})}{\partial \boldsymbol{H}} \stackrel{\circ}{\boldsymbol{H}}\right)\right\} \hat{\boldsymbol{s}},
\end{aligned}
$$

where

$$
\begin{aligned}
& \tilde{\boldsymbol{\sigma}} \equiv \boldsymbol{\sigma}-\boldsymbol{s}, \\
& F^{\prime} \equiv \frac{d F}{d H}, \\
& \hat{\boldsymbol{s}} \equiv \boldsymbol{s}-\boldsymbol{\alpha} .
\end{aligned}
$$

$c_{s}$ is a material constant.

Assume the associated flow rule and then the plastic stretching is given as

$$
D^{p}=\lambda \bar{N}(\lambda>0),
$$

where

$$
\begin{aligned}
& \lambda= \frac{\operatorname{tr}(\overline{\boldsymbol{N}} \stackrel{\circ}{\boldsymbol{\sigma}})}{\bar{M} p}, \\
& \overline{\boldsymbol{N}} \equiv \frac{\partial f(\overline{\boldsymbol{\sigma}}, \boldsymbol{H})}{\partial \boldsymbol{\sigma}} /\left\|\frac{\partial f(\overline{\boldsymbol{\sigma}}, \boldsymbol{H})}{\partial \boldsymbol{\sigma}}\right\|(\|\overline{\boldsymbol{N}}\|=1), \\
& \bar{M} p \equiv \operatorname{tr}\left[\overline { \boldsymbol { N } } \left(\overline{\boldsymbol{a}}+\left\{\frac{F^{\prime}}{F}\right.\right.\right. \\
&\left.\left.\left.-\frac{1}{R F} \operatorname{tr}\left(\frac{\partial f(\overline{\boldsymbol{\sigma}}, \boldsymbol{H})}{\partial \boldsymbol{H}} \boldsymbol{h}\right)+\frac{U}{R}\right\} \overline{\boldsymbol{\sigma}}\right)\right] .
\end{aligned}
$$

$h, \boldsymbol{h}$ and $\overline{\boldsymbol{a}}$ are functions of the stress, plastic internal state variables and $\bar{N}$ in degree one, which are related to $\dot{H}$, $\stackrel{\leftrightarrow}{\boldsymbol{H}}$ and $\stackrel{\circ}{\boldsymbol{\alpha}}$ by

$$
\begin{aligned}
& h \equiv \frac{\dot{H}}{\lambda}, \boldsymbol{h} \equiv \frac{\stackrel{\circ}{\boldsymbol{H}}}{\lambda}, \\
& \overline{\boldsymbol{a}} \equiv \frac{\frac{\circ}{\boldsymbol{\alpha}}}{\lambda}=\boldsymbol{z}-U \hat{\boldsymbol{s}}-R(\boldsymbol{z}-\boldsymbol{a}), \\
& \boldsymbol{a} \equiv \frac{\stackrel{\circ}{\boldsymbol{\alpha}}}{\lambda}, \\
& \boldsymbol{z} \equiv \frac{\stackrel{\circ}{\lambda}}{\lambda}=c_{s} \frac{\tilde{\boldsymbol{\sigma}}}{R}+\boldsymbol{a}+\frac{1}{F}\left\{F^{\prime} h-\operatorname{tr}\left(\frac{\partial f(\hat{\boldsymbol{s}}, \boldsymbol{H})}{\partial \boldsymbol{H}} \boldsymbol{h}\right)\right\} \hat{\boldsymbol{s}},
\end{aligned}
$$

since these rate variables include $\lambda$ in degree one.

The plastic stretching (16) is obtained by substituting the associated flow rule into the extended consistency condition obtained by incorporating the evolution rule (11) of the normal-yield ratio $R$ into the time-differentiation of Eq. (6) for the subloading surface. Then, the plastic loading process develops gradually as the stress approaches the normal-yield surface, exhibiting a smooth elastic-plastic transition. Thus, the

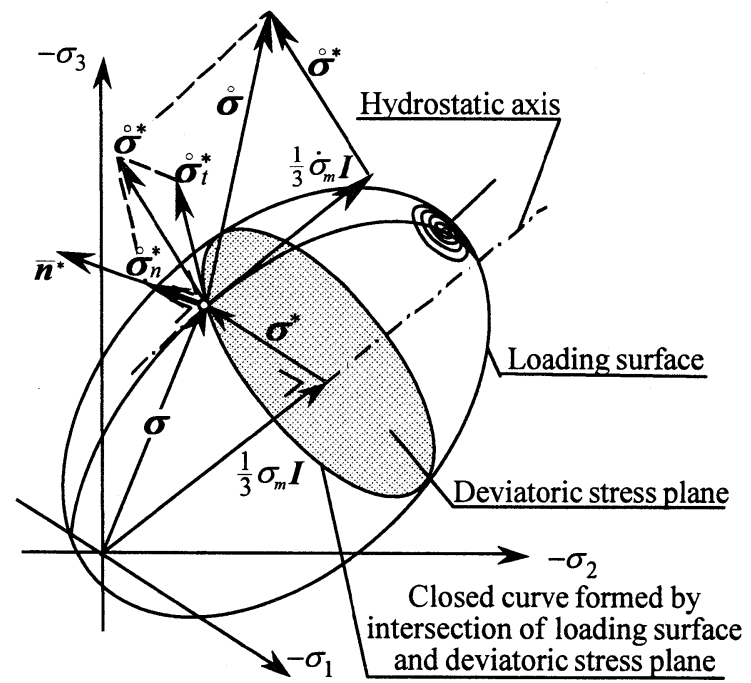

Fig. 2. Deviatoric tangential stress rate $\stackrel{\circ}{\sigma}_{t}^{*}$ illustrated in the principal stress space

subloading surface model fulfills the smoothness condition ${ }^{10)}$ 11), 12)

\subsection{Extension to tangential stress rate effect}

Let the stretching $\boldsymbol{D}$ be additively decomposed into the elastic stretching $D^{e}$ and the inelastic stretching $D^{i}$ which is further decomposed into the plastic stretching $D^{p}$ given by Eq. (16) of the subloading surface model and the additional stretching $D^{t}$, i.e.

$$
\begin{aligned}
& \boldsymbol{D}=\boldsymbol{D}^{e}+\boldsymbol{D}^{i}, \\
& \boldsymbol{D}^{i}=\boldsymbol{D}^{p}+\boldsymbol{D}^{t},
\end{aligned}
$$

where $D^{t}$ is called the tangential stretching and induced by the stress rate component tangential to the subloading surface, called the tangential stress rate, obeying the Rudnicki and Rice's conclusion" that "no vertex can result from hydrostatic stress increments" based on the consideration of the sliding mechanism in the fissure model.

The tangential stretching $D^{t}$ is formulated as

$$
\boldsymbol{D}^{t}=\frac{1}{T} \stackrel{\circ}{\boldsymbol{\sigma}}_{t}^{*},
$$

where $T$ is a monotonically decreasing function of $R$, called the tangential inelastic modulus, and simply given by

$$
T=\frac{\xi}{R^{b}} \text {. }
$$

$b(\geq 1)$ is a material constant. The second-order tensor $\stackrel{\circ}{\sigma}_{t}^{*}$, named the deviatoric tangential stress rate, is given as follows:

$$
\begin{aligned}
& \stackrel{\circ}{\boldsymbol{\sigma}}_{t} \equiv \stackrel{\circ}{\boldsymbol{\sigma}}^{*}-\stackrel{\circ}{\boldsymbol{\sigma}}_{n}^{*}, \quad \stackrel{\circ}{\boldsymbol{\sigma}}_{n}^{*} \equiv \operatorname{tr}\left(\overline{\boldsymbol{n}}^{*} \stackrel{\circ}{\boldsymbol{\sigma}}^{*}\right) \overline{\boldsymbol{n}}^{*}, \\
& \stackrel{\circ}{\boldsymbol{\sigma}}^{*} \equiv \stackrel{\circ}{\boldsymbol{\sigma}}-\dot{\sigma}_{m} \boldsymbol{I}, \quad \sigma_{m} \equiv \frac{1}{3} \operatorname{tr} \boldsymbol{\sigma},
\end{aligned}
$$




$$
\overline{\boldsymbol{n}}^{*} \equiv\left(\frac{\partial f(\overline{\boldsymbol{\sigma}}, \boldsymbol{H})}{\partial \boldsymbol{\sigma}}\right)^{*} /\left\|\left(\frac{\partial f(\overline{\boldsymbol{\sigma}}, \boldsymbol{H})}{\partial \boldsymbol{\sigma}}\right)^{*}\right\|=\frac{\overline{\boldsymbol{N}}^{*}}{\left\|\overline{\boldsymbol{N}}^{*}\right\|} .
$$

()$^{*}$ stands for the deviatoric component, and $\boldsymbol{I}$ and $\overline{\boldsymbol{n}}^{*}$ are the identity tensor and the normalized deviatoric outward-normal tensor of the subloading surface, respectively.

The deviatoric tangential stress rate $\stackrel{\circ}{\sigma}_{t}^{*}$ in the principal stress space is directed toward the tangential line of the closed curve formed by the intersection of the subloading surface and the deviatoric stress plane as illustrated in Fig. 2.

Here, it should be noted that the other models ${ }^{6)}$, 7) 8) incorporating the inelastic stretching induced by the tangential stress rate have defects as follows:

(1) These models are not applicable to materials with an arbitrary yield surface in the principal stress space since their novel stress rates inducing the additional inelastic stretching are not tangential to an arbitrary yield surface.

(2) These models are applicable only to near proportional loading process since the continuity condition is violated for the stress rate along the yield surface enclosing a purely elastic domain.

The stretching $\boldsymbol{D}$ is given from Eqs.(2), (16), (25) and (26) as

$$
\boldsymbol{D}=\boldsymbol{E}^{-1} \stackrel{\circ}{\boldsymbol{\sigma}}+\frac{\operatorname{tr}(\overline{\boldsymbol{N}} \stackrel{\circ}{\boldsymbol{\sigma}})}{\bar{M} p} \overline{\boldsymbol{N}}+\frac{1}{T} \dot{\boldsymbol{\sigma}}_{t}^{*} .
$$

The elastic modulus tensor $E$ is given by the Hooke's type as

$$
E_{i j k l}=\left(K-\frac{2}{3} G\right) \delta_{i j} \delta_{k l}+G\left(\delta_{i k} \delta_{j l}+\delta_{i l} \delta_{j k}\right),
$$

where $K$ and $G$ are elastic bulk and shear moduli, respectively, which are functions of the stress and internal state variables in general and $\delta_{i j}$ is the Kronecker's delta, i.e. $\delta_{i j}=1$ for $i=j$ and $\delta_{i j}=0$ for $i \neq j$. The inverse expression of Eq. (31) is given as:

$$
\begin{aligned}
\stackrel{\circ}{\boldsymbol{\sigma}}= & \frac{1}{1+2 G / T}\left\{\boldsymbol{E D}-\frac{\operatorname{tr}(\overline{\boldsymbol{N}} \boldsymbol{E} \boldsymbol{D})}{\bar{M}_{p}+\operatorname{tr}(\overline{\boldsymbol{N}} \boldsymbol{E} \overline{\boldsymbol{N}})}[\boldsymbol{E} \overline{\boldsymbol{N}}\right. \\
& \left.+\frac{2 G}{T}\left\{\frac{1}{3} \operatorname{tr}(\boldsymbol{E} \overline{\boldsymbol{N}}) \boldsymbol{I}-\left(\bar{M} p+\frac{1}{3} \operatorname{tr} \overline{\boldsymbol{N}} \operatorname{tr}(\boldsymbol{E} \overline{\boldsymbol{N}})\right) \frac{\overline{\boldsymbol{n}}^{*}}{\left\|\overline{\boldsymbol{N}}^{*}\right\|}\right\}\right] \\
& \left.+\frac{2 G}{T} \operatorname{tr}(\boldsymbol{E D})\left(\frac{1}{3} \boldsymbol{I}-\frac{1}{3} \operatorname{tr} \overline{\boldsymbol{N}} \frac{\overline{\boldsymbol{n}}^{*}}{\left\|\overline{\boldsymbol{N}}^{*}\right\|}\right)\right\} .
\end{aligned}
$$

Both the plastic stretching $\boldsymbol{D}^{p}$ and the tangential stretching $\boldsymbol{D}^{t}$ are formulated so as to be gradually induced as the normal-yield ratio $R$ approaches closely unity, i.e. as the stress approaches closely the normal-yield surface, exhibiting the smooth elastic-inelastic transition and fulfilling the smoothness condition and the continuity condition $\left.\left.{ }^{9)}, 10\right), 11\right), 12$. On the other hand, the extended flow models ${ }^{6)}$ 7), 8) incorporating the tangential stretching within the conventional elastoplasticity violate these conditions. Here, note that the tangential stretching $\boldsymbol{D}^{t}$ adopted for these models ${ }^{5,6,6,7,8)}$ is linearly related to the stress rate without a loading criterion,

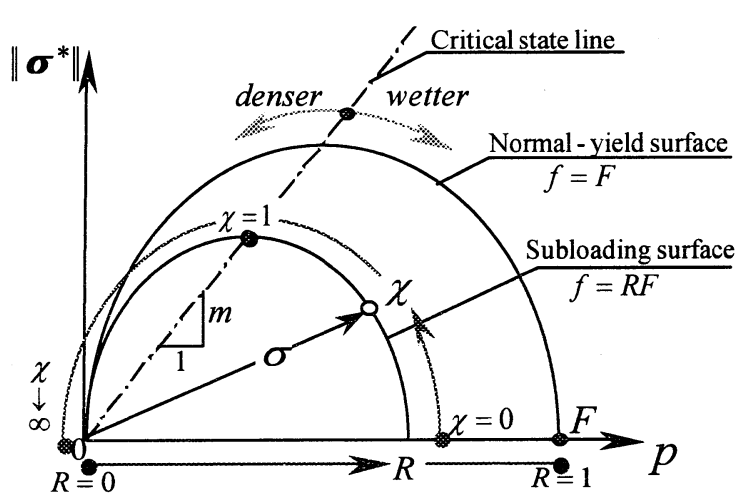

Fig. 3. The normal-y ield and the subloading surfaces in $\left(p,\left\|\boldsymbol{\sigma}^{*}\right\|\right)$ plane

and then it diminishes during an infinitesimal stress cycle, resulting in the so-called workless stretching during that cycle. For a finite stress cycle, however, the tangential stretching $D^{t}$ leads to an irreversible deformation with the loading path dependency since the total differential equation is not completely integrable leading to the violation of the exact differential form. Furthermore, these models can only describe the dependence of the direction of the inelastic stretching to that of the deviatoric stress rate component tangential to the loading surface, and then the extension to let have not only the hydrostatic stress rate dependency but also the loading criterion for the tangential stretching might be required.

The positive proportionality factor in the associated flow rule (16) is expressed in terms of the stretching $D$, rewriting $\lambda$ by $\Lambda$, as follows:

$$
\Lambda=\frac{\operatorname{tr}(\bar{N} E D)}{\bar{M}_{p}+\operatorname{tr}(\bar{N} E \bar{N})},
$$

because of $\operatorname{tr}\left(\bar{N} E \dot{\sigma}_{t}^{*}\right)=0$ for Eq.(32). Then, let the loading criterion be given by the positiveness of the proportionality factor $A$ as follows ${ }^{12)}$ :

$$
\left.\begin{array}{l}
\boldsymbol{D}^{p} \neq \boldsymbol{0}: \Lambda>0, \\
\boldsymbol{D}^{p}=\boldsymbol{0}: \Lambda \leq 0 .
\end{array}\right\}
$$

\subsection{Constitutive Equation for Isotropic Soils}

In order to focus our consideration on the effect of the tangential stretching for the stress rate response in the next chapter, the simple isotropic constitutive relation is assumed. Then, consider the case that the similarity-center of the normal-yield and the subloading surfaces is fixed in the origin of the stress space, leading to $\boldsymbol{\alpha}=\boldsymbol{s}=\boldsymbol{O}(\overline{\boldsymbol{\alpha}}=\boldsymbol{H}=\boldsymbol{O})$ (see Fig. 3). Hereinafter, let $\boldsymbol{\sigma}$ be meant the effective stress excluded the pore-pressure from the stress.

Now, the subloading surface for simple isotropic soils is given as follows:

$$
f(\boldsymbol{\sigma}, \boldsymbol{H})=p\left(1+\chi^{2}\right),
$$


where

$$
\begin{aligned}
& p \equiv-\sigma_{m}, \chi \equiv\|\boldsymbol{\eta}\| / m, \\
& \eta \equiv \boldsymbol{\sigma}^{*} / p, \boldsymbol{\sigma}^{*} \equiv \boldsymbol{\sigma}+p \boldsymbol{I} .
\end{aligned}
$$

$m$ is the material constant describing the stress ratio $\|\eta\|$ in the critical state line.

The isotropic hardening/softening function ${ }^{25)} F$ is given by

$$
F=F_{0} \exp \left(\frac{H}{\rho-\gamma}\right) .
$$

( ) 0 stands for the initial value. $\rho$ and $\gamma$ are material constants describing the slopes of the normal-consolidation and the swelling curve, respectively, in the $(\ln v, \ln p)$ plane ( $v$ : volume, $p$ : pressure).

The evolution rule of the isotropic hardening/softening variable $H$ is given by

$$
\dot{H}=-\operatorname{tr} \boldsymbol{D}^{p} \text {. }
$$

Considering that the stiffness would often be reduced not only with the increase of the stress ratio but also with the decrease of the confining pressure, the function $\xi$ in the tangential inelastic modulus $T$ of Eq. (27) is assumed as

$$
\xi=\frac{p}{a \chi^{c}} \text {. }
$$

$a$ and $c(\geq 1)$ are material constants. Then, the tangential inelastic modulus $T$ in Eq. (26) with Eqs. (27) and (41) is formulated to induce the tangential stress rate effect gradually with the increase of $\chi$ and $R$, whilst the effect decreases with the increase of pressure $p$. On the other hand, the equation of Yatomi et al. ${ }^{7}$ for the tangential inelastic modulus, i.e. $T=C(m-\|\boldsymbol{\eta}\|) p \quad(C$ : material constant $)$ is applicable only to the normal-yield state $R=1$ under the lower stress ratio than that in the critical state, i.e. $\chi \leq 1$.

Elastic bulk and shear moduli are given as

$K=\frac{p}{\gamma}, G=\frac{3(1-2 v)}{2(1+v)} K$,

where $v$ is Poisson's ratio.

\section{Mechanical Response}

The mechanical response of the present constitutive model with tangential stretching and its differences from the conventional model are examined by calculating the stress rate response to the imposed stretching in not only hardening but also softening states. Note again that the mechanical response of the model for metals exhibiting only hardening behavior was examined previously by calculating the stretching response to the imposed stress rates ${ }^{5}$.

The stress-strain relation depicted in Fig. 4, which is typical one exhibiting the softening behavior and observed in over-consolidated soils, is calculated by the subloading surface model for the drained triaxial compression with a constant lateral stress by selecting the initial values and the material

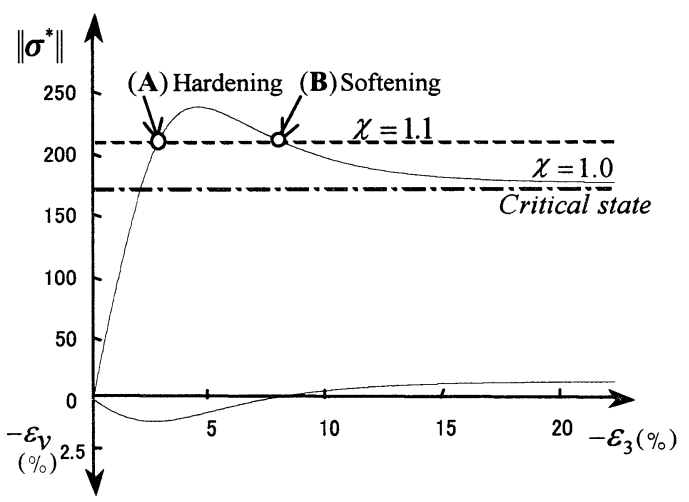

Fig. 4. The calculated result under drained triaxial compression with constant lateral pressure

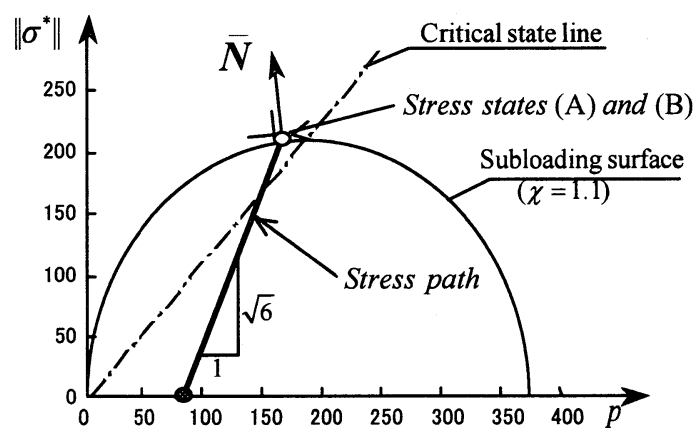

Fig. 5. The stress states (A) and (B) in $\left(p,\left\|\sigma^{*}\right\|\right)$ plane

constants as follows:

$$
\begin{gathered}
\sigma_{0}=-80.0 I, F_{0}=500, u_{R}=30, \\
\rho=0.03, \gamma=0.007, \quad m=1.16, v=0.3, \\
a=0.02, b=2.0, c=1.0 .
\end{gathered}
$$

In Fig. $4 \varepsilon_{3}$ and $\varepsilon_{v}$ are the axial strain and the volumetric strain, respectively. The tangential stretching is not induced in that loading process since the deviatoric tangential stress rate is not generated. Now, let the stretchings with various directions and the identical magnitude be imposed at the same statc of stress in the hardening (A) and the softening (B) states of Figs. 4 and 5, whilst the normal-yield ratio $R$ are 0.63 and 0.95 for (A) and (B), respectively. The stress rate $\stackrel{\circ}{\sigma}$ can be additively decomposed into the elastic stress rate $\dot{\sigma}^{e}$ and the inelastic-relaxation stress rate $\stackrel{\circ}{\sigma}^{i}$ which is further decomposed into the plastic-relaxation stress rate $\stackrel{\circ}{\sigma}^{p}$ and the tangential inelastic-relaxation stress rate $\stackrel{\circ}{\sigma}^{t}$, i.e.

$$
\begin{aligned}
& \stackrel{\circ}{\boldsymbol{\sigma}}=\stackrel{\circ}{\boldsymbol{\sigma}}^{e}+\stackrel{\circ}{\boldsymbol{\sigma}}^{i}, \\
& \stackrel{\circ}{\boldsymbol{\sigma}}^{i}=\stackrel{\circ}{\boldsymbol{\sigma}}^{p}+\stackrel{\circ}{\boldsymbol{\sigma}}^{t},
\end{aligned}
$$

where these stress rates can be derived from Eqs. (2), (24) and (25) as follows:

$$
\stackrel{\circ}{\boldsymbol{\sigma}}^{e} \equiv \boldsymbol{E D}, \quad \stackrel{\circ}{\boldsymbol{\sigma}}^{i} \equiv-\boldsymbol{E} \boldsymbol{D}^{i}, \quad \stackrel{\circ}{\boldsymbol{\sigma}}^{p} \equiv-\boldsymbol{E} \boldsymbol{D}^{p}, \quad \stackrel{\circ}{\boldsymbol{\sigma}}^{t} \equiv-\boldsymbol{E} \boldsymbol{D}^{t} .
$$

The response envelopes could be formed by connecting the 

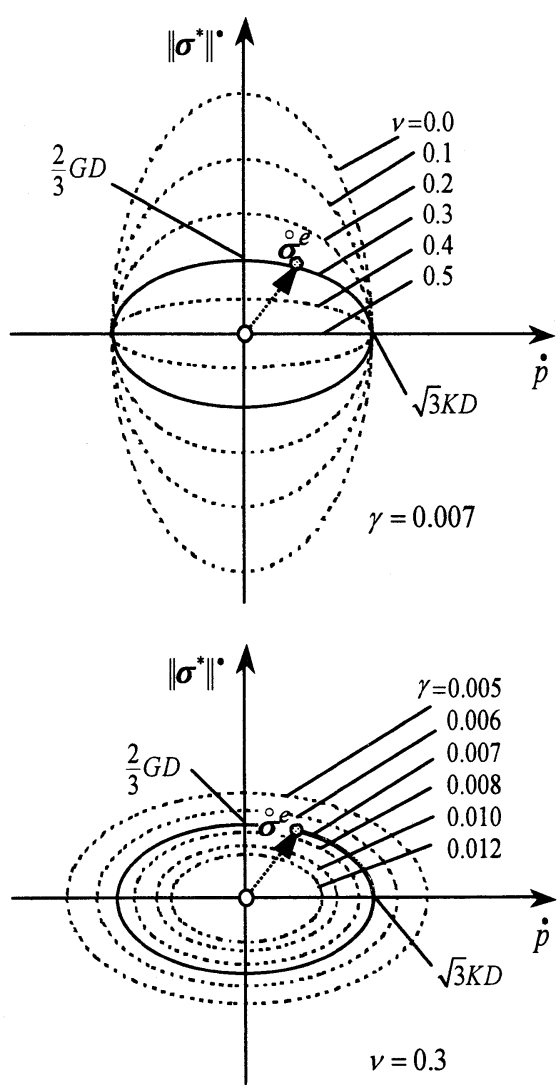

Fig. 6. The elastic stress rate envelops in $\left(\dot{p},\left\|\boldsymbol{\sigma}^{*}\right\|^{\bullet}\right)$ plane $\left(\stackrel{\circ}{\boldsymbol{\sigma}}=\dot{\circ}^{e}\right)$

end points of stress rate response vectors for the rotation of the direction of the imposed stretching $\boldsymbol{D}$ of identical magnitude in the stretching space, i.e. $\|\boldsymbol{D}\|=D=$ const. In Fig. 6 the response envelopes of the elastic stress rate $\stackrel{\circ}{ }^{e}$ to the imposed stretching of constant magnitude $D$ inducing the axisymmetric deformation are depicted for six levels of the material parameters $v$ and $\gamma$, whilst these envelopes cut the vertical and horizontal axis at $2 G D / 3$ and $\sqrt{3} K D$, respectively, in the $\left(\dot{p},\left\|\boldsymbol{\sigma}^{*}\right\|^{\bullet}\right)$ plane. Then it is shown that the response envelopes of the elastic stress rate $\dot{\circ}^{e}$ become larger as the material parameters $v$ and $\gamma$ decrease, leading to the increase of the elastic bulk and shear moduli, and especially for the case $v=0.5$ the envelopes yields a pair of straight lines. For the subsequent analysis $v=0.3$ and $\gamma=0.007$, used for the calculation in Fig. 4, are adopted.

The envelopes of the stress rate $\stackrel{\circ}{\sigma}$ to the imposed stretching $\boldsymbol{D}$ inducing the axisymmetric deformation are shown in Fig. 7 where the tangential stretching effect is not induced since the tangential stress rate does not have a deviatoric component. Here, note that the plastic-relaxation stress rate $\stackrel{\circ}{\boldsymbol{\sigma}}^{p}$ is not directed towards the inward-normal direction $-\overline{\boldsymbol{N}}$ but is directed towards $-\overline{\boldsymbol{N}}-\{(K / 2 G)$

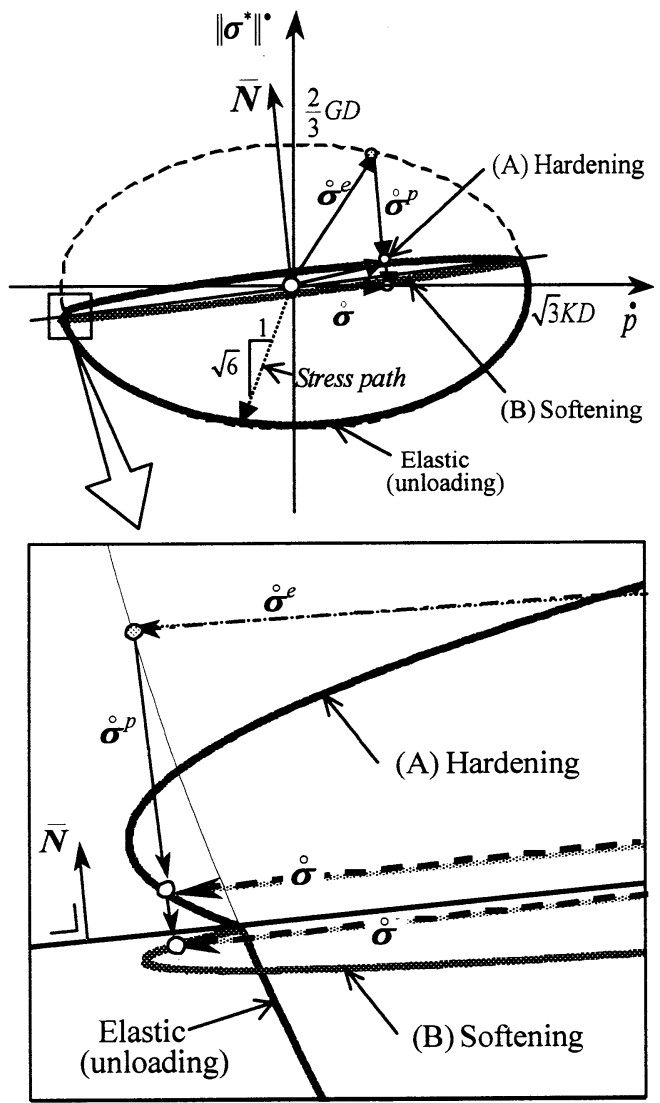

Fig. 7. The elasto-plastic stress rate envelops in $\left(\dot{p},\left\|\boldsymbol{\sigma}^{*}\right\|^{\bullet}\right)$ plane

$\left(\stackrel{\circ}{\boldsymbol{\sigma}}=\stackrel{\circ}{\boldsymbol{\sigma}}^{e}+\stackrel{\circ}{\boldsymbol{\sigma}}^{p}, \stackrel{\circ}{\boldsymbol{\sigma}}^{t}=0\right)$

$-1 / 3\}(\operatorname{tr} \overline{\boldsymbol{N}}) \boldsymbol{I}(=-\boldsymbol{E} \overline{\boldsymbol{N}} / 2 G)$. In other words, the direction of the plastic-relaxation stress rate deviates from the inward-normal direction of the subloading surface for plastically-compressible materials, i.e. $\operatorname{tr} \overline{\mathbf{N}} \neq 0$ and also for the material with the parameter $v \neq 0.0$ derived from $K / 2 G-1 / 3 \neq 0$ with Eq. (42). Therefore, they generally exhibit a small nose, whilst the prediction of the occurrence of the nose in the response envelope of the stress rate by the non-associated flow rule has been indicated as the peculiar response ${ }^{9), 18), 26), 27)}$. That is, the nose is caused not only by the non-associativity but also by the plastic compressibility, and then the impertinence of the model could not be judged by the occurrence of the nose in the stress rate responses. Further, the response envelope in the softening state exhibits the crescent form, since the stress relaxation is not induced for the stretching causing the stress rate tangential to the subloading surface but a large stress relaxation into the inward direction of the subloading surface is induced for the stretching causing the stress rate having the component normal to the subloading surface. The crescent form seems peculiar, however, and thus it would require experimental evidence and physical considerations in order to clarify its validity. 


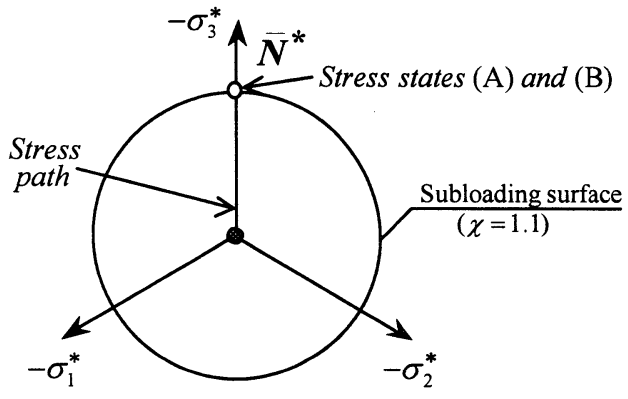

Fig. 8. The stress states (A) and (B) in the deviatoric principal stress plane

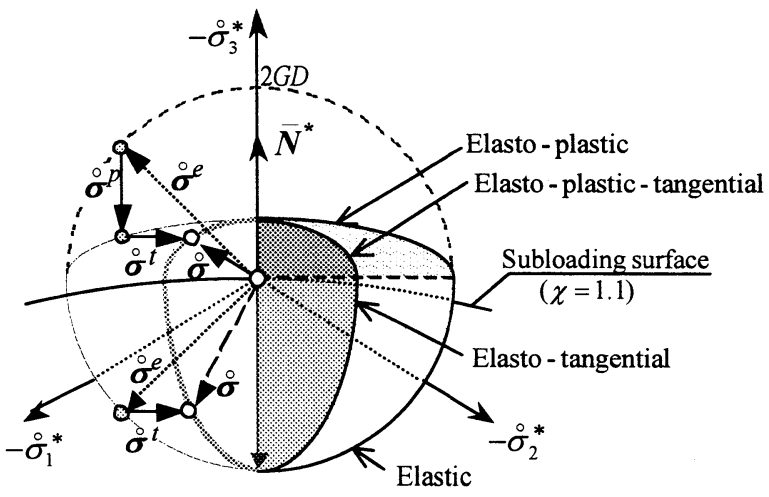

(A) Hardening

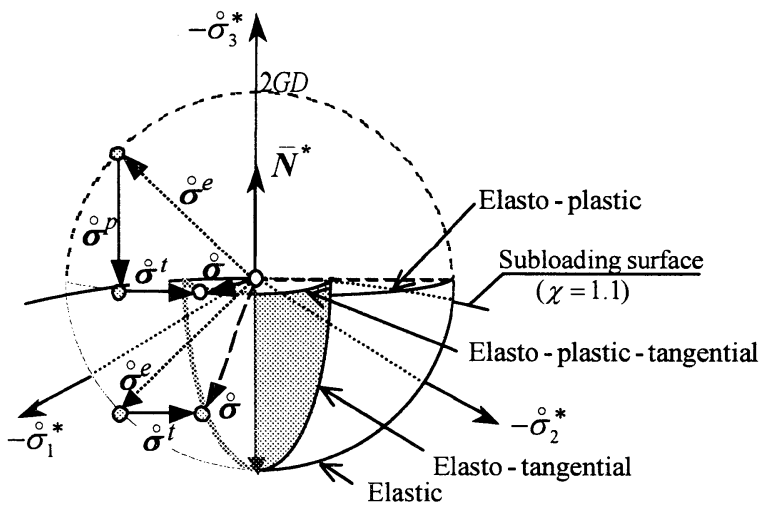

(B) Softening

Fig. 9. The elasto-plastic-tangential stress rate envelops in the deviatoric principal stress rate plane

$$
\left(\stackrel{\circ}{\boldsymbol{\sigma}}=\stackrel{\circ}{\boldsymbol{\sigma}}^{e}+\stackrel{\circ}{\boldsymbol{\sigma}}^{p}+\stackrel{\circ}{\boldsymbol{\sigma}}^{t}\right)
$$

The response envelopes of the stress rate $\stackrel{\circ}{\sigma}$ to the imposed deviatoric stretching with various directions and the identical magnitude $D$ at the stress state (A) and (B) in Fig. 8 are depicted in the deviatoric stress rate plane $\left(-\stackrel{\circ}{\sigma}_{1}^{*}\right.$, $\left.-\stackrel{\circ}{\sigma}_{2}^{*},-\stackrel{\circ}{\sigma}_{3}^{*}\right)$ as shown in Fig. 9. The elastic stress rate responses $\dot{\boldsymbol{\sigma}}^{e}$ cut the each axis at $2 G D$ in the deviatoric stress rate plane for both the stress state (A) and (B). Furthermore, the plastic-relaxation stress rate $\stackrel{\circ}{\sigma}^{p}$ and the tangential inelastic-relaxation stress rate $\stackrel{\circ}{\sigma}^{t}$ have the inward-normal direction and the tangential one, respectively, of the subloading surface. Thus, the stress rate response is decreased not only for the normal direction but also for the tangential one by the tangential stretching effect. In addition, the direction of the inelastic-relaxation stress rate $\stackrel{\circ}{\sigma}^{i}$ is not given uniquely at the give stress state, whilst the stress rate envelope exhibits a symmetrical form due to the adoption of the associated flow rule for the plastic stretching. Therefore, it does not exhibit a nose but exhibits again the crescent form in the softening state. Here, note that a nose is predicted by the non-associated flow rule even for the response envelope to the imposed deviatoric stretching.

\section{Concluding Remarks}

The main features of subloading surface model for soils with tangential stretching are as follows:

i ) Both the magnitude and the direction of the inelastic stretching depend on the stress rate tangential to the subloading surface.

ii) The deformation behavior for not only normal-yield but also subyield states can be described.

The stress rate response to the imposed stretching predicted by the present model has the following characteristics.

i ) The response envelope of the stress rate to the imposed stretching exhibits a small nose in $\left(\dot{p},\left\|\sigma^{*}\right\|^{\bullet}\right)$ plane for which the tangential stress rate effect is not induced. The nose is caused by the plastic compressibility.

ii) Due to the tangential stretching effect the response envelope of the stress rate in the deviatoric stress rate plane $\left(-\stackrel{\circ}{\sigma}_{1}^{*}\right.$, $\left.-\stackrel{\circ}{\sigma}_{2}^{*},-\stackrel{\circ}{\sigma}_{3}^{*}\right)$ is decreased for the tangential direction of the subloading surface.

iii) The response envelope to the imposed deviatoric stretching in the deviatoric stress rate plane does not exhibit any nose. On the other hand, a nose is predicted by the non-associated flow rule not only for the response envelope of the stress rate in the $\left(\dot{p},\left\|\sigma^{*}\right\|^{\bullet}\right)$ plane but also for that in the deviatoric stress rate plane.

iv) The response envelope in the softening state exhibits a crescent form despite of the tangential stress rate effect.

These responses seem to be peculiar. Hereafter, the essentiality of them would have to be examined by both physical considerations and experiments.

\section{Acknowledgment}

The authors would like to express our sincere gratitude to Prof. C. Yatomi, Dept. Civil Eng., Kanazawa University, for his valuable advices on this problem. This research was supported partly by the Research Fellowships of the Japan Society for the Promotion of Science for Young Scientists. 


\section{REFERENCES}

1) Ishihara, K. and Towhata, I., Sand response to cyclic rotation of principal stress directions as induced by wave loads. Soils and Found. Vol. 23, No. 4, pp. 11-26, 1983.

2) Pradel, D., Ishihara, K. and Gutierrez, M., Yielding and flow of sand under principal stress axes rotation. Soils and Found. Vol. 30, No. 1, pp. 87-99, 1990.

3) Gutierrez, M., Ishihara, K. and Towhata, I., Flow theory for sand during rotation of principal stress direction. Soils and Found. Vol. 31, No. 4, pp. 121-132, 1991.

4) Hashiguchi, K., The extended flow rule in plasticity. Int. J. Plasticity, Vol. 13, pp. 37-58, 1997.

5) Hashiguchi, K. and Tsutsumi, S., Elastoplastic constitutive equation with tangential stress rate effect. Int. J. Plasticity, Vol.17, pp. 117-145, 2001.

6) Rudnicki, J.W. and Rice, J.R., Conditions for localization of deformation in pressure-sensitive dilatant materials. J. Mech. Phys. Solids, Vol. 23, pp. 371-394, 1975.

7) Yatomi, C., Yashima, A., Iizuka, A. and Sano, I., General theory of shear bands formation by a non-coaxial Cam-clay model. Soils and Found. Vol. 29, No. 3, pp. 41-53, 1989.

8) Papamichos, E. and Vardoulakis, I., Shear band formation in sand according to non-coaxial plasticity model. Geotechnique, Vol. 45, pp. 649-661, 1995.

9) Hashiguchi, K., Fundamental requirements and formulation of elastoplastic constitutive equations with tangential plasticity. Int. J. Plasticity, Vol. 9, pp. 525-549, 1993.

10) Hashiguchi, K., Mechanical requirements and structures of cyclic plasticity models. Int. J. Plasticity, Vol. 9, pp. 721-748, 1993.

11) Hashiguchi, K., The extended flow rule in plasticity. Int. J. Plasticity, Vol. 13, pp. 37-58, 1997.

12) Hashiguchi, K., Fundamentals in constitutive equation: continuity and smoothness conditions and loading criterion. Soils and Found. Vol. 40, No. 4, pp. 155-161, 2000.

13) Hashiguchi, K. and Ueno, M., Elastoplastic constitutive laws of granular materials. in Constitutive Equations of Soils (Proc. 9th Int. Conf. Soil Mech. Found. Eng., Spec. Session 9, Tokyo), JSSMFE, pp. 73-82, 1977.

14) Hashiguchi, K., Constitutive equations of elastoplastic materials with elastic-plastic transition. J. Appl. Mech.
(ASME) Vol. 47, pp. 266-272, 1980.

15) Hashiguchi, K., Subloading surface model in unconventional plasticity. Int. J. Solids Struct. Vol. 25, pp. 917-945, 1989.

16) Hashiguchi, K., A generalized formulation of the concept of nonhardening region. Int. J. Plasticity Vol. 11, pp. 347-365, 1995.

17) Gudehus, G., A comparison of some constitutive laws for soils under radially symmetric loading and unloading. Proc. 3rd Int. Conf. Numer. Meth. Geomech. Aachen, Vol. 4, pp. 1309-1323, 1979.

18)Vermeer, P.V., A five-constant model unifying well-established concepts. Results of Int. Workshops on Constitutive Relations in Soils, Grenoble, pp. 175-197, 1982.

19)Dafalias, Y.F., The plastic spin. J. Appl. Mech. (ASME) Vol. 52, pp. 865-871, 1985.

20) Zbib, H.M. and Aifantis, E.C., On the concept of relative and plastic spins and its implications to large deformation theories. Part I: Hypoelasticity and vertex-type plasticity. Acta Mech. Vol. 75, pp. 15-33, 1988.

21) Hashiguchi, K. and Chen, Z.-P., Elastoplastic constitutive equation of soils with the subloading surface and the rotational hardening. Int. J. Numer. Anal. Meth. Geomech. Vol. 22, pp. 197-227, 1998.

22) Drucker, D.C., Conventional and unconventional plastic response and representation. Appl. Mech. Rev. (ASME) Vol. 41, pp. 151-167, 1988.

23) Masing, G., Eigensppannungen und Verfestigung beim Messing. Proc. 2nd Int. Congr. Appl. Mech., Zurich, pp. 332-335, 1926.

24) Mroz, Z., On forms of constitutive laws for elastic-plastic solids. Archiwum Mechaniki Stosowanej, Vol. 18, pp. 1-34, 1966.

25) Hashiguchi, K., Isotropic hardening theory of granular media. Proc. JSCE, Vol. 227, pp. 45-60, 1974.

26) Molenkamp, F. and Van Ommen, A., Peculiarity of non-associativity in plasticity of soil mechanics. Int. J. Numer. Anal. Meth. Geomech. Vol. 11, pp. 659-661, 1987.

27) Hashiguchi, K., Inexpedience of the non-associated flow rule. Int. J. Numer. Anal. Meth. Geomech. Vol. 15, pp. 753-756, 1991. 